\title{
Influence of different playing surfaces on bone mass accretion in male adolescent football players: a one-season study.
}

\begin{tabular}{|c|c|}
\hline Journal: & Part P: Journal of Sports Engineering and Technology \\
\hline Manuscript ID & JSET-18-0076.R3 \\
\hline Manuscript Type: & Original Article \\
\hline $\begin{array}{r}\text { Date Submitted by the } \\
\text { Author: }\end{array}$ & $n / a$ \\
\hline Complete List of Authors: & $\begin{array}{l}\text { Lozano-Berges, Gabriel; GENUD (Growth, Exercise, NUtrition and } \\
\text { Development) Research Group, Universidad de Zaragoza; Faculty of } \\
\text { Health and Sport Sciences, Department of Physiatry and Nursing; } \\
\text { Instituto Agroalimentario de Aragón-IA2-(Universidad de Zaragoza-CITA) } \\
\text { Matute-Llorente, Ángel; GENUD (Growth, Exercise, NUtrition and } \\
\text { Development) Research Group, Universidad de Zaragoza; Faculty of } \\
\text { Health and Sport Sciences, Department of Physiatry and Nursing; } \\
\text { Instituto Agroalimentario de Aragón-IA2-(Universidad de Zaragoza - } \\
\text { CITA); Centro de Investigación Biomédica en Red de Fisiopatología de la } \\
\text { Obesidad y Nutrición (CIBERObn) } \\
\text { Gómez-Bruton, Alejandro; GENUD (Growth, Exercise, NUtrition and } \\
\text { Development) Research Group, Universidad de Zaragoza; Faculty of } \\
\text { Health and Sport Science, Department of Physiatry and Nursing; } \\
\text { Instituto Agroalimentario de Aragón-IA2-(Universidad de Zaragoza- } \\
\text { CITA); Centro de Investigación Biomédica en Red de Fisiopatología de la } \\
\text { Obesidad y Nutrición (CIBERObn) } \\
\text { Gonzalez-Aguero, Alex; GENUD (Growth, Exercise, NUtrition and } \\
\text { Development) Research Group, Universidad de Zaragoza; Faculty of } \\
\text { Health and Sport Sciences, Department of Physiatry and Nursing; } \\
\text { Instituto Agroalimentario de Aragón-IA2-(Universidad de Zaragoza- } \\
\text { CITA); Centro de Investigación Biomédica en Red de Fisiopatología de la } \\
\text { Obesidad y Nutrición (CIBERObn) } \\
\text { Vicente-Rodríguez, Germán; GENUD (Growth, Exercise, NUtrition and } \\
\text { Development) Research Group, Universidad de Zaragoza; Faculty of } \\
\text { Health and Sport Sciences, Department of Physiatry and Nursing; } \\
\text { Instituto Agroalimentario de Aragón-IA2-(Universidad de Zaragoza- } \\
\text { CITA); Centro de Investigación Biomédica en Red de Fisiopatología de la } \\
\text { Obesidad y Nutrición (CIBERObn) } \\
\text { Casajús, José; GENUD (Growth, Exercise, NUtrition and Development) } \\
\text { Research Group, Universidad de Zaragoza; Faculty of Health Sciences, } \\
\text { Department of Physiatry and Nursing; Instituto Agroalimentario de } \\
\text { Aragón-IA2-(Universidad de Zaragoza-CITA); Centro de Investigación } \\
\text { Biomédica en Red de Fisiopatología de la Obesidad y Nutrición } \\
\text { (CIBERObn) }\end{array}$ \\
\hline Keywords: & $\begin{array}{l}\text { Soccer, body composition, bone density, bone mass, third-generation } \\
\text { artificial turf }\end{array}$ \\
\hline Abstract: & $\begin{array}{l}\text { There are different surfaces on which football is played, but their } \\
\text { influence on bone mass accretion still remains unknown. The aims of this } \\
\text { study were to compare bone mass accretion between football players } \\
\text { and controls and evaluate the influence of two different playing surfaces } \\
\text { on bone accretion. Twenty-seven male football players }(13.2 \pm 0.5 \text { y) and } \\
15 \text { controls }(12.6 \pm 1.1 \text { y) participated in this study. Football players were }\end{array}$ \\
\hline
\end{tabular}


classified into two groups according to the surface they trained on: 14 on third-generation artificial turf with elastic layer (3G-EL) and 13 on thirdgeneration artificial turf without elastic layer (3G-NEL). Bone mineral content (BMC) and areal bone mineral density (aBMD) were measured with dual-energy $\mathrm{X}$-ray absorptiometry. Bone mineral apparent density (BMAD) variables were calculated. Bone geometry and strength of the non-dominant tibia were assessed with peripheral quantitative computed tomography. For both football players and controls, bone variables measured at subtotal body, lumbar spine, legs and tibia $(p<0.05)$ significantly increased. Based on the time spent practicing football, the increase in aBMD for the legs $(p<0.05)$ was higher in football players than controls. Moreover, lumbar spine BMAD increased more in 3G-NEL players in comparison with $3 G-E L$ players $(p<0.05)$. Playing football on 3G-EL and 3G-NEL seems to positively affect bone mass during growth. After playing for one season on these playing surfaces, football practice on 3G-NEL with the lower shock absorption seems to have produced the highest increment in aBMD at lumbar spine. Thus, football practice on surfaces with lower shock absorption could provide an extra benefit on bone health.

\section{SCHOLARONE Manuscripts}


2 Influence of different playing surfaces on bone mass

3 accretion in male adolescent football players: a one-

4 season study

5 Gabriel Lozano-Berges, Ángel Matute-Llorente, Alejandro

6 Gómez-Bruton, Alex González-Agüero, Germán Vicente-

7 Rodríguez, José A. Casajús.

8 Abstract

9 There are different surfaces on which football is played, but their influence on bone

10 mass accretion still remains unknown. The aims of this study were to compare bone

11 mass accretion between football players and controls and evaluate the influence of two

12 different playing surfaces on bone accretion. Twenty-seven male football players

$13(13.2 \pm 0.5 \mathrm{y})$ and 15 controls $(12.6 \pm 1.1 \mathrm{y})$ participated in this study. Football players

14 were classified into two groups according to the surface they trained on: 14 on third-

15 generation artificial turf with elastic layer (3G-EL) and 13 on third-generation artificial

16 turf without elastic layer (3G-NEL). Bone mineral content (BMC) and areal bone

17 mineral density (aBMD) were measured with dual-energy X-ray absorptiometry. Bone

18 mineral apparent density (BMAD) variables were calculated. Bone geometry and

19 strength of the non-dominant tibia were assessed with peripheral quantitative computed

20 tomography. For both football players and controls, bone variables measured at subtotal

21 body, lumbar spine, legs and tibia $(p<0.05)$ significantly increased. Based on the time

22 spent practicing football, the increase in aBMD for the legs $(p<0.05)$ was higher in

23 football players than controls. Moreover, lumbar spine BMAD increased more in 3G-

24 NEL players in comparison with $3 \mathrm{G}-\mathrm{EL}$ players $(p<0.05)$. Playing football on $3 \mathrm{G}-\mathrm{EL}$

25 and 3G-NEL seems to positively affect bone mass during growth. After playing for one

26 season on these playing surfaces, football practice on 3G-NEL with the lower shock

27 absorption seems to have produced the highest increment in aBMD at lumbar spine.

28 Thus, football practice on surfaces with lower shock absorption could provide an extra

29 benefit on bone health.

30 Keywords

31 Soccer, body composition, bone density, bone mass, third-generation artificial turf

32

33 


\section{Introduction}

35 Childhood and adolescence are crucial periods for bone building and children should reduce the risk of having low bone mass ${ }^{1}$ by means of physical exercise and sports participation. ${ }^{2}$ In fact, a recent review by Weaver et al. ${ }^{3}$ graded the positive effects of physical activity on bone mass with a grade A (maximum level of evidence). However, the review did not include the effects of individual sports. A study by Mautalen ${ }^{4}$ highlighted the positive effects of football practice on bone mass during adolescent growth. These positive effects on bone mass are mainly explained by the fact that football is a weight-bearing sport which is characterized by high-impact actions, such as accelerations, decelerations, changes of direction, jumps and kicks. ${ }^{5}$ Furthermore, this sport has great importance for young people because football is one of the most, if not the most, practiced sport worldwide. ${ }^{6}$

The positive benefits of football practice on bone tissue have been amply demonstrated $;{ }^{7}$ higher levels of bone mineral content (BMC) or areal bone mineral density (aBMD) levels when compared to a control group (CG) have been reported in youth football players. ${ }^{8-10}$ More importantly, the positive effects generated by football have been shown to remain after 1- and 3-year follow-ups. ${ }^{8,11-15}$ To assess bone mass, most of studies performed on youth football teams have used dual-energy X-ray absorptiometry (DXA). Although DXA is capable of explaining up to $60 \%$ of the variance in bone strength, it cannot directly measure bone geometry variables. ${ }^{16}$ For this reason, some studies have used other techniques, such as hip structural analysis (HSA) ${ }^{14}$ and peripheral quantitative computed tomography (pQCT) ${ }^{17-19}$ for measuring bone geometry in football players. HSA is derived from hip scan images acquired by DXA. According to the International Society for Clinical Densitometry (ISCD), the hip is not the recommended site for evaluating BMC and aBMD in children and adolescents due to its high variability during bone development. ${ }^{20}$ Thus, bone geometry measured with HSA in young populations could be biased as described above. In contrast, pQCT, which is not influenced by bone size like DXA, measures trabecular and cortical bone, allowing for evaluation of the tibia, which is directly affected by football. Up to now, only one study has compared young male football players and the CG, showing higher bone geometry in football players than the CG. ${ }^{19}$ On the other hand, to the authors' knowledge, there are no studies which have evaluated the effects of playing football compared to a population not engaged in any sport on bone geometry and strength measured by pQCT during growth.

Ground reaction forces could be described as one of the main contributing factors influencing bone accretion. However, properties of playing surfaces change over time, which may affect this relationship. The number of natural grass football fields is decreasing, whereas the number of artificial turf pitches is increasing. ${ }^{21}$ To replicate the playing properties of natural grass football fields, new developments in construction methods of artificial turf fields include the use of materials such as rubber and sand infill. ${ }^{21}$ At the same time, different infill materials create different mechanical characteristics. ${ }^{22}$ Thus, the inclusion or lack of an elastic layer in the installation process generates differences in shock absorption and vertical deformation forces. ${ }^{22}$ Due to these mechanical properties, different types of surfaces may evoke different loads to the bone. To the authors' knowledge, only Plaza-Carmona et al. ${ }^{23}$ compared the influence of third-generation artificial turf fields and soil football fields on bone mass accrual in male children football players, finding no differences for BMC and aBMD. However, the influence of recent third-generation artificial turfs with an elastic layer (3G-EL) and without an elastic layer (3G-NEL) on bone tissue in young football players is yet 
unknown. During adolescent growth, the short-term effects on bone mass while playing

84

85

86

87

88

89

90

91

92

93

94

95

96

\section{Methods} bone values. study field).

football versus not playing a sport should be studied more deeply, especially bone geometry and strength. Therefore, the aims of this study were: 1) to compare BMC, aBMD, bone mineral apparent density (BMAD), bone geometry and bone strength between young male football players and the CG; and 2) to evaluate the influence of training and playing football on two playing surfaces (3G-EL or 3G-NEL) on previous

The authors hypothesized that all adolescents will improve bone mass and strength values throughout the season, but football players will have increased bone mass, geometry and strength compared to the CG. Also, those football players who play on 3G-NEL during this period will exhibit additional bone mass gain in comparison with players on the $3 \mathrm{G}$-EL due to the fact that the $3 \mathrm{G}-\mathrm{NEL}$ surface will have lower shock absorption than the 3G-EL. Therefore, the football players will receive increased loads.

\section{Participants}

Two football clubs and two high schools in Aragon (Spain) were invited to participate. Although 35 football players and 23 controls agreed to participate, 16 participants were excluded because of the following reasons: three football players and three controls did not perform the second measurement citation, four football players and two controls did not wear the accelerometer, and one football player and three controls had blurred DXA or pQCT images. Consequently, the final sample of 27 male football players (13.17 \pm 0.52 years) and 15 male controls (CG; $12.58 \pm 1.11$ years) participated in this study (Fig. 1). Football players were split into two groups according to the surface where they trained and played: 3G-EL ( $\mathrm{n}=14 ; 13.01 \pm 0.61$ years) and 3G-NEL $(\mathrm{n}=13$; $13.35 \pm 0.34$ years). Although the $C G$ were physically active, they were not regularly engaged in any sport. Measurements were performed in Zaragoza (Spain) at the beginning of the season (October-December 2013) and end of the season (May-July 2014) with a mean measurement time of $31.5 \pm 6.2$ weeks, which followed the protocol recommended by the ISCD ${ }^{20}$ to evaluate bone changes between DXA scans at a minimum interval of six months.

The years of exposure to football practice prior to the beginning of this study were $5 \pm 2$ years in $3 \mathrm{G}$-EL players and $5 \pm 1$ years in 3G-NEL players. Hours of training per week were individually quantified based on the number of training sessions in which the player participated (3G-EL players $=2.6 \pm 0.2$ hours/week; 3G-NEL players $=2.3 \pm 0.3$ hours/week). A sport scientist monitored the type of exercises performed by each team during their training throughout the season. Each training session lasted approximately $90 \mathrm{~min}$, including 5-min warm-up consisting of low-intensity running; 5-10 min of lowintensity games; $60 \mathrm{~min}$ of technical football exercises (passing, kicking, running, dribbling); and 5-10 minutes of cool down stretching exercises. Taking into account training sessions and matches played at home and away grounds, the percentage of time spent on surfaces included in the present study were $80.2 \%$ for $3 \mathrm{G}-\mathrm{EL}$ players and $78.1 \%$ for $3 \mathrm{G}-\mathrm{NEL}$ players (i.e. away games reduced the percentages of per time on the

Participants, parents and coaches of each club were informed about the protocol, and the possible benefits and risks associated with this study. Written informed consent from parents and verbal assent from the participants were obtained. This study was performed in accordance with the Declaration of Helsinki 1961 (revision of Fortaleza 
2013). The protocol was approved by the Ethics Committee of Clinical Research from

132 the Government of Aragon (CEICA, Spain) [C.I. PI13/0091]. The research was

133 registered in a public database Clinicaltrials.gov [NCT02399553]. This longitudinal

134 study is part of a larger randomized controlled trial that evaluated the effect of football

135 surfaces and boot model on bone mass and strength in male and female adolescent

136 football players. However, female football players were not included in the present manuscript because of the low number of participants that could be evaluated in the second assessment (some players stopped playing and others did not perform to the evaluation). The Transparent Reporting of Evaluations with Nonrandomized Designs (TREND) Statement was used as a guideline for reporting non-randomized trials. ${ }^{24}$

\section{Anthropometric measurements}

Height (stadiometer SECA 225, SECA, Hamburg, Germany; to the nearest $0.1 \mathrm{~cm}$ ) and weight (scale SECA 861, SECA, Hamburg, Germany; to the nearest $0.1 \mathrm{~kg}$ ) were measured without shoes and with minimum clothing. Body mass index (BMI) was calculated by dividing the participant's weight $(\mathrm{kg})$ by the squared height $\left(\mathrm{m}^{2}\right)$.

\section{Maturity status}

Pubertal maturity was determined according to the stages proposed by Tanner and Whitehouse ${ }^{25}$ and using a self-assessment method which has been shown to be a valid and reliable technique. ${ }^{26}$

\section{Calcium intake}

Milligrams of daily calcium intake were calculated by a validated calcium food frequency questionnaire. ${ }^{27}$ Participants were asked how many times per day, week or months they consumed calcium-rich foods.

\section{Physical activity measurements}

Physical activity was assessed with triaxial accelerometers (GENEActiv developed by Unilever Discover, Colworth, UK; and distributed by ActivInsights Ltd., Kimbolton, Cambridge, UK). These accelerometers have been calibrated and validated for measuring physical activity in children and adolescents on different body locations, including the right and the left wrists. ${ }^{28}$ All participants wore GENEA devices on their non-dominant wrist for seven days, except for football players who had to remove the accelerometer during official matches. Data were recorded at $30 \mathrm{~Hz}$ and analysed at 1-s epochs. These data were taken within a duration of seven days towards the end of the season (May-July 2014). Accelerometer data were analysed using the software RStudio (version RStudio Desktop 1.0.153, Boston, MA, United States). Minutes of valid time 
175 in light, moderate and vigorous physical activity, as well as sedentary time, were

176 calculated using cut-points proposed by Phillips et al. ${ }^{28}$ for right wrist as follows: light,

$1772.4-7.9 \mathrm{~g} \cdot \mathrm{s}$; moderate, $8.0-21.0 \mathrm{~g} \cdot \mathrm{s}$; vigorous $>21.1 \mathrm{~g} \cdot \mathrm{s}$; sedentary, $<2.3 \mathrm{~g} \cdot \mathrm{s}$; and for

178 left wrist as follows: light, $2.7-7.1 \mathrm{~g} \cdot \mathrm{s}$; moderate, $7.2-22.5 \mathrm{~g} \cdot \mathrm{s}$; vigorous $>22.6 \mathrm{~g} \cdot \mathrm{s}$;

179 sedentary, $<2.6 \mathrm{~g} \cdot \mathrm{s}$.

180

\section{Bone measurements}

\section{DXA}

183 Bone and lean masses were measured with DXA QDR-Explorer (pediatric version of

184 the software QDR-Explorer, Hologic Corp. Software version 12.4, Bedford, MA, USA). DXA equipment was calibrated daily following the manufacturer guidelines. Whole body, non-dominant hip and lumbar spine scans were conducted with participants in supine position by the same technician who had been fully been trained to perform the

188 scans. The positioning of the subjects and analysis of the results were performed

189 according to the manufacturer's guidelines. The non-dominant limb was determined by 190 asking which leg would be used to kick a ball. ${ }^{29}$

191

192

193

194

195

\section{3 pQCT}

214 Bone mass, geometry and strength were measured at the non-dominant tibia using a

215 Stratec XCT-2000 L pQCT scanner (Stratec Medizintechnik, Pforzheim, Germany).

216 This device is a rotate-translate scanner that obtains a trans-axial image. Following the 217 guidelines provided by the manufacturer, the pQCT calibration was performed daily 218 using a quality control phantom. Coefficients of variation for each pQCT variable used 219 in the present study were as follows: $0.71 \%$ for total volumetric bone mineral density 220 (Tt.vBMD), $0.93 \%$ for trabecular vBMD (Tb.vBMD), $0.25 \%$ for total BMC (Tt.BMC), $2210.25 \%$ for cortical vBMD (Ct.vBMD), $0.73 \%$ for cortical cross sectional area (Ct.Ar), 
$1.12 \%$ for cortical thickness (Ct.Th), $2.51 \%$ for fracture load in X-axis (Frc.LdX); and $2.08 \%$ for polar strength strain index (SSIp).

Tibia length was determined as the inner border of the medial condyle to the farthest point of the medial malleolus of the tibia and it was always measured by the same technician using a wooden ruler (to the nearest $1 \mathrm{~mm}$ ). Then, the non-dominant leg was centred in the imaging field and the foot and knee were secured to reduce movement. The scanner was positioned on the distal tibia, and a scout view was performed to manually set the reference line on the midpoint of the distal tibia end plate. Bone parameters were assessed at 4\% (distal tibia) and 38\% (diaphyseal tibia) of the length of the tibia with a voxel dimension of $0.5 \mathrm{~mm}$ and a slice thickness of $1 \mathrm{~mm}$. Following $\mathrm{ISCD}^{34}$ recommendations for evaluating bone geometry and strength with pQCT, Tt.vBMD $\left(\mathrm{mg} / \mathrm{cm}^{3}\right)$ and Tb.vBMD $\left(\mathrm{mg} / \mathrm{cm}^{3}\right)$ at the $4 \%$ site of the tibia were analysed. Moreover, the parameters measured at $38 \%$ of the length of the tibia were total Tt.BMC (g), Ct.vBMD (mg/cm $\left.{ }^{3}\right)$, Ct.Ar $\left(\mathrm{mm}^{2}\right)$, Ct.Th (mm), Frc.LdX (N) and SSIp $\left(\mathrm{mm}^{3}\right)$.

All pQCT images were analysed with version 6.20 of the manufacturer's software. Contour mode 1 with a threshold of $180 \mathrm{mg} / \mathrm{cm}^{3}$ for the $4 \%$ site of the tibia and 280 $\mathrm{mg} / \mathrm{cm}^{3}$ for the $38 \%$ site of the tibia was used to determine the periosteal surface of the bone. At $4 \%$ site of the tibia, trabecular bone was determined from a central area covering $45 \%$ of the total bone cross-sectional area. At $38 \%$ site of the tibia, cortical bone was obtained using cortical mode 1 with a threshold of $710 \mathrm{mg} / \mathrm{cm}^{3}$. Additionally, cortical mode 1 with a threshold of $280 \mathrm{mg} / \mathrm{cm}^{3}$ was used to obtain bone strength variables (Frc.LdX and SSIp). After that, bone mineralization of $1200 \mathrm{mg} / \mathrm{cm}^{3}$ was assumed.

\section{Mechanical properties of the pitches}

Two different surfaces were included in the present study: 3G-EL and 3G-NEL. By the time the study was performed, no more than six years had passed since they were installed. Both pitches presented similar infill characteristics and were constructed by the same manufacturer.

Assessments of mechanical characteristics of the football fields used in the present study were performed according to the quality standards proposed by the European Committee for Standardisation (EN 15530-1:2007) and the Handbook of Test Methods for Football Turf. ${ }^{35}$ This standard is applied for amateur, educational and recreational sport and evaluates the performance and durability of outdoor sport surfaces. Thus, test requirements used for evaluating mechanical properties of the football pitches are as follows: ball rebound has to be between 0.608 and $1.012 \mathrm{~m}$; ball roll between 4 and 10 m; shock absorption between 55 and 70\%; vertical deformation between 4 and $10 \mathrm{~mm}$; and rotational resistance between 25 and $50 \mathrm{~N} \cdot \mathrm{m}$. Although maintenance of these football fields could vary, both $3 \mathrm{G}-\mathrm{EL}$ (ball rebound: $0.825 \mathrm{~m}$; ball roll: $10 \mathrm{~m}$; shock absorption: $62 \%$; vertical deformation: $7 \mathrm{~mm}$; and rotational resistance: $50 \mathrm{~N} \cdot \mathrm{m}$ ) and 3G-NEL (ball rebound: $0.944 \mathrm{~m}$; ball roll: $10 \mathrm{~m}$; shock absorption: $56 \%$; vertical deformation: $6 \mathrm{~mm}$; and rotational resistance: $41 \mathrm{~N} \cdot \mathrm{m}$ ) were within these parameters.

These mechanical characteristics were measured in five field positions following the quality standards guidelines. Each test was performed three or five times (according to the required attempts) in all field positions. An Advanced Artificial Athlete was used for measuring shock absorption and vertical deformation variables. The other mechanical characteristics were measured with the equipment proposed by the 
All tests were performed at stable meteorological conditions at temperatures between 10 and $22^{\circ} \mathrm{C}$, wind speed between 0 and $1.2 \mathrm{~m} / \mathrm{s}$ and humidity between 45 and $60 \%$.

272 Pocket Weather Tracker 4000 (Kestrelmeters, Birmingham, UK) was used to evaluate

273 meteorological conditions. These measurements were performed in April (3G-NEL) and 274 May (3G-EL).

\section{Statistical analyses}

\section{Sample size calculation}

278 To the authors knowledge, there have been no studies that have calculated whole body 279 or lumbar spine BMAD. Therefore, data from a Zouch et al. ${ }^{13}$ study evaluating aBMD 280 at whole body in football players and the CG $\left(1.098 \pm 0.093\right.$ and $1.010 \pm 0.087 \mathrm{~g} / \mathrm{cm}^{2}$, 281 respectively) was used to calculate sample size. Due to the fact that the main analysis of 282 the present study was the repeated measures, the sample size calculation was performed 283 for these analyses. The sample size for repeated measures was calculated in whole body 284 aBMD to get a power of $80 \%$ at the $5 \%$ alpha level and to reject the null hypothesis $285 \mathrm{H}_{0}: \mu 1=\mu 2$. Thus, assuming a small to medium effect size $(f=0.20)$ and a correlation 286 among repeated measures of 0.7 at pre- and post-season, a total sample size of 32 (16 287 per group) would be needed.

288

\section{Outcome measures treatment}

290 Statistical Package for the Social Sciences (SPSS) version 22.0 for Mac OS X (SPSS

291 Inc., Chicago, IL, USA) was used for the statistical analyses. All variables showed 292 normal distribution tested with the Kolmogorov-Smirnov test.

293 Chi-square test was performed to evaluate differences between pubertal stages.

294 Independent t-tests were applied to examine differences among groups for descriptive 295 characteristics and bone parameters at pre- and post-season. ANOVA for repeated 296 measures were applied to check differences within all football players and the CG, as 297 well as within 3G-EL and 3G-NEL between pre- and post-season without adjusting by 298 covariates (Model 1). After that, these analyses were repeated, including two covariates 299 as follow: Model $1+$ minutes per day of moderate-vigorous physical activity (MVPA; 300 Model 2); and Model $2+$ total lean mass less head for DXA parameters or tibia muscle 301 area for pQCT parameters (Model 3). MVPA was selected as a covariate to analyse the 302 possible effect of these high intensity activities on bone mass. In addition, lean mass 303 was used as a covariate due to its influence on bone mass. ${ }^{36}$ Moreover, weight was not 304 used as covariate to avoid multicollinearity in the analysis due to its high correlation 305 with lean mass (Pearson correlation coefficient $=0.922 ; p<0.001$ ). Group by time 306 interactions for changes in bone values were also performed by repeated measures 307 analyses.

308 Effect size statistics using Cohen's $d$ was calculated for independent t-test and partial 309 eta squared $\left(\eta_{\mathrm{p}}^{2}\right)$ for repeated measures analyses. The effect size for Cohen's $\mathrm{d}$ can be 310 small $(0.2-0.5)$, medium $(0.5-0.8)$ or large $(>0.8)$ and partial eta squared $\left(\eta^{2} \mathrm{p}\right)$ can be 311 small $(0.01-0.06)$, medium $(0.06-0.14)$ or large $(>0.14)$. Statistical significance was 312 set at $p<0.05$.

\section{Results}

315 Descriptive data 
The physical characteristics of the participants are shown in Table 1. No differences were found in any descriptive data between football players and controls (Cohen's $d$ ranged from 0.05 to $0.69 ; p>0.05$ ). Between different surfaces (3G-EL and 3G-NEL), no differences were found either (Cohen's $d$ ranged from 0.06 to $0.70 ; p>0.05$ ).

As expected, there were significant differences for the age, weight, height, BMI, subtotal lean mass, tibia length and tibia muscle area between pre- and post-season in all groups $\left(\eta_{p}^{2}\right.$ ranged from $<0.001$ to $0.943 ; p<0.05$ ). Moreover, football players who trained on $3 \mathrm{G}-\mathrm{NEL}$ demonstrated lower percentage of body fat at post- than pre-season $\left(\eta_{\mathrm{p}}^{2}\right.$ was $\left.0.357 ; p<0.05\right)$.

No significant differences were found in MVPA between football players and the CG (93.29 \pm 19.93 vs $95.50 \pm 33.46$ minutes per day; $95 \% \mathrm{CI},-17.54$ to -21.95 ; Cohen's $d$ was $0.08 ; p>0.05)$ and between football players who trained in $3 \mathrm{G}-\mathrm{EL}$ and $3 \mathrm{G}-\mathrm{NEL}$ $(100.25 \pm 21.69$ vs $85.80 \pm 15.29$ minutes per day; $95 \%$ CI, -29.29 to 0.40 ; Cohen's $d$ was $0.77 ; p>0.05)$.

\section{$B M C, a B M D$ and $B M A D$}

\section{2}

333

334

\section{Comparisons between football players and the $\mathrm{CG}$}

Table 2 summarizes BMC and aBMD measured at pre- and post-season in football players and CG. Higher legs aBMD was found in football players than the CG at postseason $(95 \% \mathrm{CI}=-0.02-0.19$; Cohen's $d$ was $0.72 ; p<0.05)$. Football players and the CG significantly increased subtotal body BMC, lumbar spine BMC, legs aBMD and lumbar spine BMAD $\left(\eta_{\mathrm{p}}^{2}\right.$ ranged from 0.192 to $\left.0.713 ; p<0.05\right)$. Furthermore, a significant group by time interaction was found for legs aBMD (percentage changes of football players and CG were $7.0 \%$ and $4.0 \%$, respectively; $\eta_{p}^{2}$ was $0.097 ; p<0.05$ ). This interaction showed that the increase in the legs aBMD was significantly greater in football players in comparison with the CG. The same result was obtained when MVPA were included as covariate (percentage changes of football players and the CG were $7.0 \%$ and $4.1 \%$, respectively; $\eta^{2}$ p was $\left.0.099 ; p<0.05\right)$, but it became non-significant when subtotal lean mass was introduced as covariate (percentage changes of football players and the CG were $6.9 \%$ and $4.5 \%$, respectively; $\eta^{2}$ p was $0.063 ; p>0.05 ;$ Fig. 2 ). Therefore, a lean mass correction could underestimate the effects of this high-impact sport on bone mass.

\section{Comparison between 3G-EL and 3G-NEL football players}

Table 3 summarizes BMC and aBMD measured at pre- and post-season in 3G-EL and 3G-NEL football players. 3G-EL showed higher lumbar spine and femoral neck BMAD at pre- and post-season than 3G-NEL (Cohen's $d$ ranged from 0.80 to $1.45 ; p<0.05$ ). Both football groups increased subtotal body BMC, lumbar spine BMC, legs aBMD and lumbar spine BMAD from pre- to post-season $\left(\eta^{2}\right.$ ranged from 0.203 to $0.674 ; p<$ 0.05). Moreover, 3G-NEL also increased femoral neck BMAD $\left(\eta_{p}^{2}\right.$ was $0.096 ; p<$ 0.05 ). There was a group by time interaction for lumbar spine BMAD (percentage changes of $3 \mathrm{G}-\mathrm{EL}$ and $3 \mathrm{G}-\mathrm{NEL}$ players were $1.8 \%$ and $5.9 \%$, respectively; $\eta_{\mathrm{p}}^{2}$ was $0.169 ; p<0.05)$. This interaction demonstrated that during one year of football practice, BMAD at lumbar spine increased more in football players who trained on 3G-NEL than in those who trained on 3G-EL. On the other hand, no group by time interaction was found for lumbar spine BMAD when MVPA was included as covariate (percentage 
changes of 3G-EL and 3G-NEL players were $2.6 \%$ and $5.9 \%$, respectively; $\eta^{2}$ was $0.109 ; p>0.05$; Fig. 3).

364

\section{Bone geometry and strength}

\section{Comparisons between football players and the $\mathrm{CG}$}

367 Bone geometry and strength measured at the $4 \%$ and $38 \%$ sites of the tibia in football 368 players and the CG are shown in Table 2. Higher Ct.Ar at pre-season and SSIp at pre369 and post-season were found in football players than the CG (Cohen's d ranged from 3700.63 to $0.80 ; p<0.05)$. Both groups improved Tt.BMC, Ct.Ar, Ct.Th, Frc.LdX and $371 \operatorname{SSIp}\left(\eta_{\mathrm{p}}^{2}\right.$ ranged from 0.106 to $\left.0.635 ; p<0.05\right)$. Furthermore, the CG decreased 372 Tt.vBMD and Tb.vBMD at distal tibia $\left(\eta^{2}\right.$ were 0.102 and $\left.0.128 ; p<0.05\right)$. No group 373 by time interactions were found in bone geometry and strength values $\left(\eta_{\mathrm{p}}^{2}\right.$ ranged from $374<0.001$ to $0.053 ; p<0.05$ ); however, when tibia muscle area was added as covariate, 375 there was a group by time interaction for Tt.BMC (percentage changes of football 376 players and the CG were $5.7 \%$ and $8.1 \%$, respectively; $\eta^{2}$ was $0.105 ; p<0.05$; Figure 377 2). This interaction demonstrated that Tt.BMC increased more in the CG than football 378 players. As determined in DXA parameters, a tibia muscle area correction could modify 379 the differences between groups and, consequently, under-estimate the effects of this 380 high-impact sport on bone mass.

381

382 Comparison between $3 \mathrm{G}-\mathrm{EL}$ and $3 \mathrm{G}-\mathrm{NEL}$ football players

383 Bone geometry and strength measured at the $4 \%$ and $38 \%$ sites of the tibia in $3 \mathrm{G}-\mathrm{EL}$ and 3G-NEL football players are shown in Table 3. At pre- and post-season, 3G-EL players showed higher Tt.vBMD and Tb.vBMD than 3G-NEL (Cohen's $d$ ranged from 1.01 to $1.31 ; p<0.05)$. 3G-EL and 3G-NEL players improved Tt.BMC, Ct.Ar, Ct.Th, Frc.LdX and SSIp ( $\eta^{2}$ p ranged from 0.199 to $\left.0.683 ; p<0.05\right)$. There were no group by time interactions between both 3G-EL and 3G-NEL players even when MVPA and tibia muscle area were used as covariates $\left(\eta^{2}\right.$ ranged from $<0.001$ to $\left.0.086 ; p<0.05\right)$.

\section{Discussion}

The main finding of the present study is that one season of football practice, independent of the playing surface, positively affect bone accretion in the lower limbs of young players. Subtotal body BMC and lumbar spine BMAD are the variables that ISCD recommends to compare densitometry results in children and adolescents. ${ }^{20}$ However, the analysis of legs would help to explain how football practice affects bone mass because legs are the closest site of the body to the floor and support more impact than the other bone sites. On the other hand, football players playing on $3 \mathrm{G}-\mathrm{EL}$ and $3 \mathrm{G}-$ NEL demonstrated similar bone mass, geometry and strength increases in most variables studied, except for lumbar spine BMAD that increased more in football players who played on 3G-NEL than those who played on 3G-EL.

The present study has demonstrated that legs aBMD improved more in football players than the CG. To the best of the authors' knowledge, six studies have analysed the effects of football practice on DXA parameters in young football players and the CG. ${ }^{8,11-15}$ Most of them demonstrated that football practice seems to be a good strategy for increasing BMC and aBMD during growth. These results were higher for football players than those obtained by the CG. Moreover, they also reported higher BMC and 
aBMD at lower limbs in football players than the CG. Most of them also demonstrated differences in lumbar spine, a preferred site to assess densitometry variables during growth. ${ }^{20}$ Nonetheless, none of them included subtotal body site and BMAD parameters in their study. Therefore, their results could be slightly influenced by bone mass of the skull (site not responsive to physical activity and their loads ${ }^{37}$ ) and bone size of their participants. ${ }^{31}$ In the present study, although subtotal body BMC and lumbar spine BMAD were included, no significant differences in these variables were found between football players and the CG. These results could be explained by the fact that the number of hours per week of football training could not be sufficient to have significant bone differences between groups. In summary, football practice during childhood and adolescence might help to attain a higher peak of bone mass and, consequently, to reduce the risk of suffering osteoporosis during adulthood.

In terms of bone geometry and strength parameters, the present study showed that bone strength was higher in football players than the CG, with larger SSIp values. Up to now, there are only a cross-sectional study ${ }^{19}$ and a 1-year follow-up study ${ }^{14}$ that have compared bone geometry and strength values between male football players and the CG using pQCT and HSA, respectively. Despite the use of different techniques, all of them found greater, but not significant, bone geometry and strength in football players than the CG. The lack of differences between these groups could be explained by the fact that cortical bone parameters increase sharply after the age of $14^{38}$ and the age of participants of the present study was lower. Thus, future studies evaluating bone geometry and strength acquisition before and after 14 years of age will help to clarify the effects of football practice on these bone variables during growth.

To date, only a cross-sectional study performed by Plaza-Carmona et al. ${ }^{23}$ analysed bone mass in football players who trained on different playing surfaces (artificial and soil fields). These authors showed that neither BMC nor aBMD were different between football players according to playing surface. The present study demonstrated that lumbar spine BMAD, femoral neck BMAD, Tt.vBMD and Tb.vBMD were higher in 3G-EL than 3G-NEL players at both pre- and post-season. Although no significant differences in hours of trainings per week were found between football groups (3G-EL players $=2.6 \pm 0.2$ hours/week; 3G-NEL players $=2.3 \pm 0.3$ hours/week), the extra $15-20$ minutes per week of training performed by players who trained in $3 \mathrm{G}-\mathrm{EL}$ might explain the observed bone differences between both football groups. In addition to this, as demonstrated Varley et al. ${ }^{18}$, an increase of training volume improved bone geometry and strength parameters.

Artificial fields aim to emulate physical and mechanical characteristics of natural surfaces. In fact, since rubber and sand were added in artificial turf, differences in mechanical variables and the number of injuries between both surfaces were reduced. ${ }^{21}$, ${ }^{39}$ Afterwards, the inclusion of the elastic layer behind the artificial turf systems increased shock absorption, ${ }^{22}$ and consequently, reduced the amount of load received by football players. Although shock absorption characteristics measured in the present study in 3G-EL (62\%) and 3G-NEL (56\%) were different, the effects of each surface on bone mass, geometry and strength seem to be similar between fields with the exception of lumbar spine BMAD. The closest bone sites to the ground receive the highest impacts produced by football and progressively, as they move away from the ground to other bone sites, these impacts dissipate. Impacts produced in both 3G-EL and 3G-NEL at tibia and femoral neck sites are high and cause similar bone adaptations. However, only loads produced by football actions in 3G-NEL are capable of causing an extra lumbar spine BMAD compared to those produced in 3G-EL. On the other hand, the fact that both football groups included in this study trained less than three hours per week 
458 could be limiting the differences between football players. To reinforced this idea, 459 Zouch et al. ${ }^{11}$ and Varley et al. ${ }^{18}$ demonstrated that higher training volume improved 460 both DXA and pQCT parameters.

461

462

463

464

465

466

467

468

469

470

471

472

473

474

475

476

477

478

479

480

481

482

483

Prior to the beginning of this study, sample sizes of each group were higher than that obtained in the sample size calculation. Nevertheless, some participants were excluded because of the above-mentioned reasons (see Methods section). Consequently, the main limitation of the present study was that sample sizes of 3G-EL $(n=14), 3 G-N E L ~(n=$ $13)$ and the $C G(n=15)$ were lower than the predicted number obtained in the sample size calculation (16 participants per group). Therefore, bone comparisons between groups may have been affected by type II error. Moreover, the type of football exercises performed by each team was similar but not equal. Therefore, the variation in football training exercises could cause slight differences in bone mass. Participants' calcium intake data may be somewhat unreliable due to the fact that questionnaires were undertaken by the youth participants with supervision by the researchers, as opposed to their parents or guardians. Another limitation was that the number of practice hours per week for both teams was lower than those in other studies performed with football players (approximately 2.4 vs 10.0 and 11.9 hours per week). ${ }^{14,18}$ Moreover, football players could not use accelerometers during matches and accelerations registered could not accurately represent the accelerations of lower limbs as they had to be placed on the wrist. On the other hand, the main strength was that this is the first study that evaluated the influence of two third-generation artificial turf surfaces (3G-EL and 3G-NEL) on bone mass, geometry and strength in male adolescent football players. Moreover, this is also the first study that compares bone geometry and strength between football players and the CG.

\section{Conclusions}

485

486

487

488

489

490

\section{Acknowledgements}

The authors want to thank all the children, their parents and football clubs (C.D.

\section{Funding}

497 This work was supported by the Spanish "Ministerio de Economia y Competitividad" 498 (Project DEP 2012-32724)'. GLB received a Grant FPU 2013 (FPU13/02111) from the 499 "Ministerio de Educación, Cultura y Deporte".

500

\section{Declaration of conflicting interests}

502 The authors declare that there is no conflict of interest. 


\section{References}

504

505

506

507

508

509

510

511

512

513

514

515

516

517

518

519

520

521

522

523

524

525

526

527

528

529

530

531

532

533

534

535

536

537

538

539

540

541

542

543

544

545

546

547

548

549

550

551

1. Rizzoli R, Bianchi ML, Garabedian M, et al. Maximizing bone mineral mass gain during growth for the prevention of fractures in the adolescents and the elderly.

Bone 2010; 46: 294-305.

2. Tenforde AS and Fredericson M. Influence of sports participation on bone health in the young athlete: a review of the literature. $P m R$ 2011; 3: 861-867.

3. Weaver CM, Gordon CM, Janz KF, et al. The National Osteoporosis Foundation's position statement on peak bone mass development and lifestyle factors: a systematic review and implementation recommendations. Osteoporos Int 2016; 27 : 1281-1386.

4. Mautalen CA. Soccer and bone development. Osteoporos Int 2016; 27: 3133-3134.

5. Bangsbo J. The physiology of soccer with special reference to intense intermittent exercise. Acta Physiol Scand Suppl 1994; 619: 1-155.

6. Kunz M. 265 million playing football. FIFA magazine. July 2007, p. 11-15.

7. Lozano-Berges G, Matute-Llorente Á, González-Agüero A, et al. Soccer helps build strong bones during growth: a systematic review and meta-analysis. Eur $J$ Pediatr 2018; 177: 295-310.

8. Vicente-Rodriguez G, Ara I, Perez-Gomez J, et al. High femoral bone mineral density accretion in prepubertal soccer players. Med Sci Sports Exerc 2004; 36: 1789-1795.

9. Nebigh A, Rebai H, Elloumi M, et al. Bone mineral density of young boy soccer players at different pubertal stages: relationships with hormonal concentration. Joint Bone Spine 2009; 76: 63-69.

10. Seabra A, Marques E, Brito J, et al. Muscle strength and soccer practice as major determinants of bone mineral density in adolescents. Joint Bone Spine 2012; 79: 403-408.

11. Zouch M, Jaffre C, Thomas T, et al. Long-term soccer practice increases bone mineral content gain in prepubescent boys. Joint Bone Spine 2008; 75: 41-49.

12. Zouch M, Vico L, Frere D, et al. Young male soccer players exhibit additional bone mineral acquisition during the peripubertal period: 1-year longitudinal study. Eur $J$ Pediatr 2013; 173: 53-61.

13. Zouch M, Zribi A, Alexandre C, et al. Soccer Increases Bone Mass in Prepubescent Boys During Growth: A 3-Yr Longitudinal Study. J Clin Densitom 2015; 18: 179186.

14. Vlachopoulos D, Barker AR, Ubago-Guisado E, et al. Longitudinal Adaptations of Bone Mass, Geometry, and Metabolism in Adolescent Male Athletes: The PROBONE Study. J Bone Miner Res 2017; 32: 2269-2277.

15. Vlachopoulos D, Barker AR, Ubago-Guisado E, et al. The effect of 12-month participation in osteogenic and non-osteogenic sports on bone development in adolescent male athletes. The PRO-BONE study. J Sci Med Sport 2018; 21: 404409.

16. Sievanen H. A physical model for dual-energy X-ray absorptiometry--derived bone mineral density. Invest Radiol 2000; 35: 325-330.

17. Anliker E, Sonderegger A and Toigo M. Side-to-side differences in the lower leg muscle-bone unit in male soccer players. Med Sci Sports Exerc 2013; 45: 15451552.

18. Varley I, Hughes DC, Greeves JP, et al. Increased Training Volume Improves Bone Density and Cortical Area in Adolescent Football Players. Int J Sports Med 2017; 38: 341-346. 
19. Lozano-Berges G, Matute-Llorente A, Gomez-Bruton A, et al. Bone geometry in young male and female football players: a peripheral quantitative computed tomography (pQCT) study. Arch Osteoporos 2018; 13: 57.

20. Crabtree NJ, Arabi A, Bachrach LK, et al. Dual-energy X-ray absorptiometry interpretation and reporting in children and adolescents: the revised 2013 ISCD Pediatric Official Positions. J Clin Densitom 2014; 17: 225-242.

21. McNitt AS. Synthetic turf in the USA - Trends and issues. ITSRJ 2005; 10: 27-33.

22. Burillo P, Gallardo L, Felipe JL, et al. Mechanical assessment of artificial turf football pitches: The consequences of no quality certification. Sci Res Essays 2012; 7: 2457-2465.

23. Plaza-Carmona MP, Vicente-Rodriguez G, Martin-Garcia M, et al. Influence of hard vs. soft ground surfaces on bone accretion in prepubertal footballers. Int $J$ Sports Med 2014; 35: 55-61.

24. Des Jarlais DC, Lyles C, Crepaz N, et al. Improving the reporting quality of nonrandomized evaluations of behavioral and public health interventions: the TREND statement. Am J Public Health 2004; 94: 361-366.

25. Tanner JM and Whitehouse RH. Clinical longitudinal standards for height, weight, height velocity, weight velocity, and stages of puberty. Arch Dis Child 1976; 51: 170-179.

26. Duke PM, Litt IF and Gross RT. Adolescents' self-assessment of sexual maturation. Pediatrics 1980; 66: 918-920.

27. Julián Almárcegui C, Huybrechts I, Gómez Bruton A, et al. Validity of foodfrequency questionnaire for estimating calcium intake in adolescent swimmers. Nutr Hosp 2015; 32: 1773-1779.

28. Phillips LR, Parfitt G and Rowlands AV. Calibration of the GENEA accelerometer for assessment of physical activity intensity in children. J Sci Med Sport 2013; 16: 124-128.

29. Hoffman M, Schrader J, Applegate T, et al. Unilateral postural control of the functionally dominant and nondominant extremities of healthy subjects. $J$ Athl Train 1998; 33: 319-322.

30. Gracia-Marco L, Ortega FB, Jimenez-Pavon D, et al. Adiposity and bone health in Spanish adolescents. The HELENA study. Osteoporos Int 2012; 23: 937-947.

31. Carter DR, Bouxsein ML and Marcus R. New approaches for interpreting projected bone densitometry data. J Bone Miner Res 1992; 7: 137-145.

32. Katzman DK, Bachrach LK, Carter DR, et al. Clinical and anthropometric correlates of bone mineral acquisition in healthy adolescent girls. $J$ Clin Endocrinol Metab 1991; 73: 1332-1339.

33. Gomez-Bruton A, Gonzalez-Aguero A, Casajus JA, et al. Swimming training repercussion on metabolic and structural bone development; benefits of the incorporation of whole body vibration or pilometric training; the RENACIMIENTO project. Nutr Hosp 2014; 30: 399-409.

34. Adams JE, Engelke K, Zemel BS, et al. Quantitative computer tomography in children and adolescents: the 2013 ISCD Pediatric Official Positions. J Clin Densitom 2014; 17: 258-274.

35. FIFA. FIFA Quality concept for football turf. Handbook of requirements, Fédération Internationale de Football Association, October 2015.

36. Vicente-Rodriguez G, Urzanqui A, Mesana MI, et al. Physical fitness effect on bone mass is mediated by the independent association between lean mass and bone mass through adolescence: a cross-sectional study. J Bone Miner Metab 2008; 26 : 288-294. 
602 37. Taylor A, Konrad PT, Norman ME, et al. Total body bone mineral density in young 603 children: influence of head bone mineral density. J Bone Miner Res 1997; 12: 652604655.

605

606

607

38. Binkley TL, Specker BL and Wittig TA. Centile curves for bone densitometry measurements in healthy males and females ages 5-22 yr. J Clin Densitom 2002; 5: 343-353.

608 39. Dragoo JL and Braun HJ. The effect of playing surface on injury rate: a review of 609 the current literature. Sports Med 2010; 40: 981-990. 
Table 1. Subject characteristics of football players who played in different surfaces and controls. Pre-season moment

\section{Post-season moment} All players $\begin{array}{ccc}(\mathrm{n}=27) & \mathrm{CG} & \text { All players } \\ (\mathrm{n}=15) & (\mathrm{n}=27)\end{array}$ Age (year)

10 Weight $(\mathrm{kg})$ $13.17 \pm 0.52$

Height $(\mathrm{cm})$
BMI $\left(\mathrm{kg} \cdot \mathrm{m}^{-2}\right)$ $50.57 \pm 11.19$ $158.32 \pm 8.77$

Daily calcium intake $(\mathrm{mg})$

$158.32 \pm 8.77$
$19.99 \pm 3.06$

$819.88 \pm 210.85$

$34269.71 \pm 6804.01$

$23.98 \pm 6.86$

$12.58 \pm 1.11$
$46.26 \pm 8.94$

$(\mathrm{n}=27)$

$\begin{array}{cc}46.26 \pm 8.94 & 54.07 \pm 12.09^{*} \\ 153.37 \pm 8.82 & 162.97 \pm 9.11^{*}\end{array}$

$162.97 \pm 9.11^{*}$

$793.18 \pm 309.33$

Subtotal lean mass $(\mathrm{g})$

$\quad 855.09+329.62$

$855.09 \pm 329.62$

$30965.97 \pm 5982.53$

$7438.26 \pm 7871.54 *$
$22.50 \pm 6.59 *$

$24.35 \pm 6.76$

$22.50 \pm 6.5$

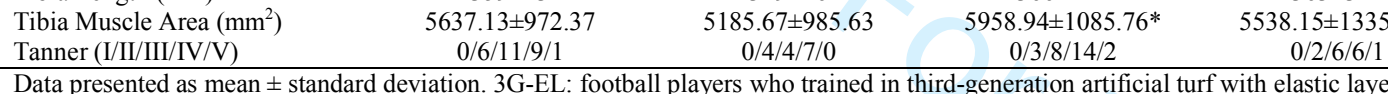

$5958.94 \pm 1085.76$

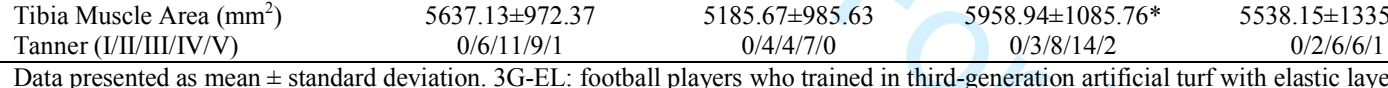

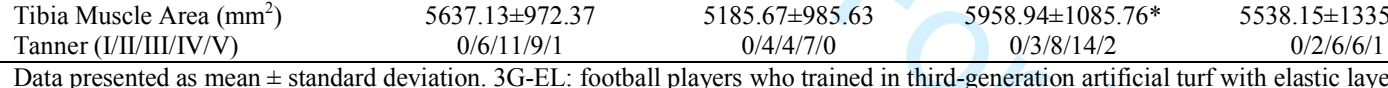

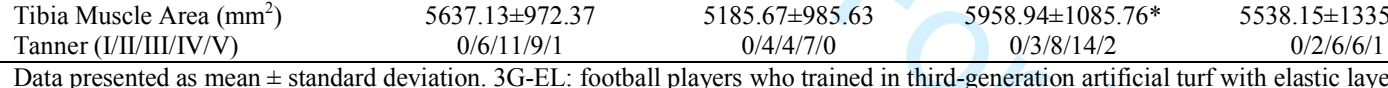

CG

CG
$(\mathrm{n}=15)$

$13.37 \pm 1.15^{*}$

$50.32 \pm 9.77 *$

$158.62 \pm 9.15^{*}$

$1001.54 \pm 505.92$

$34295.15 \pm 7183.19^{*}$

$23.92+6.61$

$23.92 \pm 6.6$
$363+32 *$

$5538.15 \pm 1335.06^{*}$

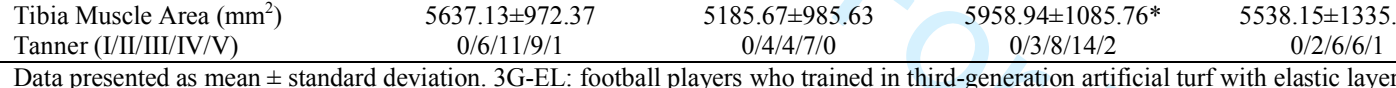

\begin{tabular}{cc} 
& Pre-season m \\
& $3 \mathrm{G}-\mathrm{EL}$ \\
$(\mathrm{n}=14)$ \\
\hline$*$ & $13.01 \pm 0.61$ \\
$*$ & $50.89 \pm 12.01$ \\
$5^{*}$ & $157.26 \pm 10.09$ \\
$*$ & $20.35 \pm 3.24$ \\
.92 & $812.27 \pm 213.77$ \\
$3.19 *$ & $34677.22 \pm 7597.57$ \\
& $23.57 \pm 7.53$ \\
& $357 \pm 25$ \\
$.06 *$ & $5823.79 \pm 1066.85$
\end{tabular}

on momen

3G-NEL $(\mathrm{n}=13)$ $50.23 \pm 10.72$

$159.47 \pm 7.31$

$19.60 \pm 2.93$

$828.08 \pm 216.06$

$5823.79 \pm 1066.85$
$0 / 3 / 7 / 3 / 1$

$33830.86 \pm 6113.04$

$24.42 \pm 6.34$

$362 \pm 21$

$5436.12 \pm 855.09$
$0 / 3 / 4 / 6 / 0$

3G-EL

Post-season moment

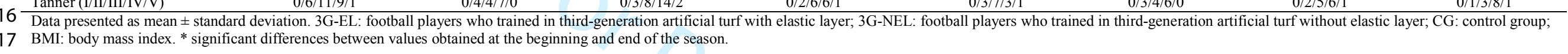


Table 2. Bone mineral content, density, strength and structure in football players and controls.

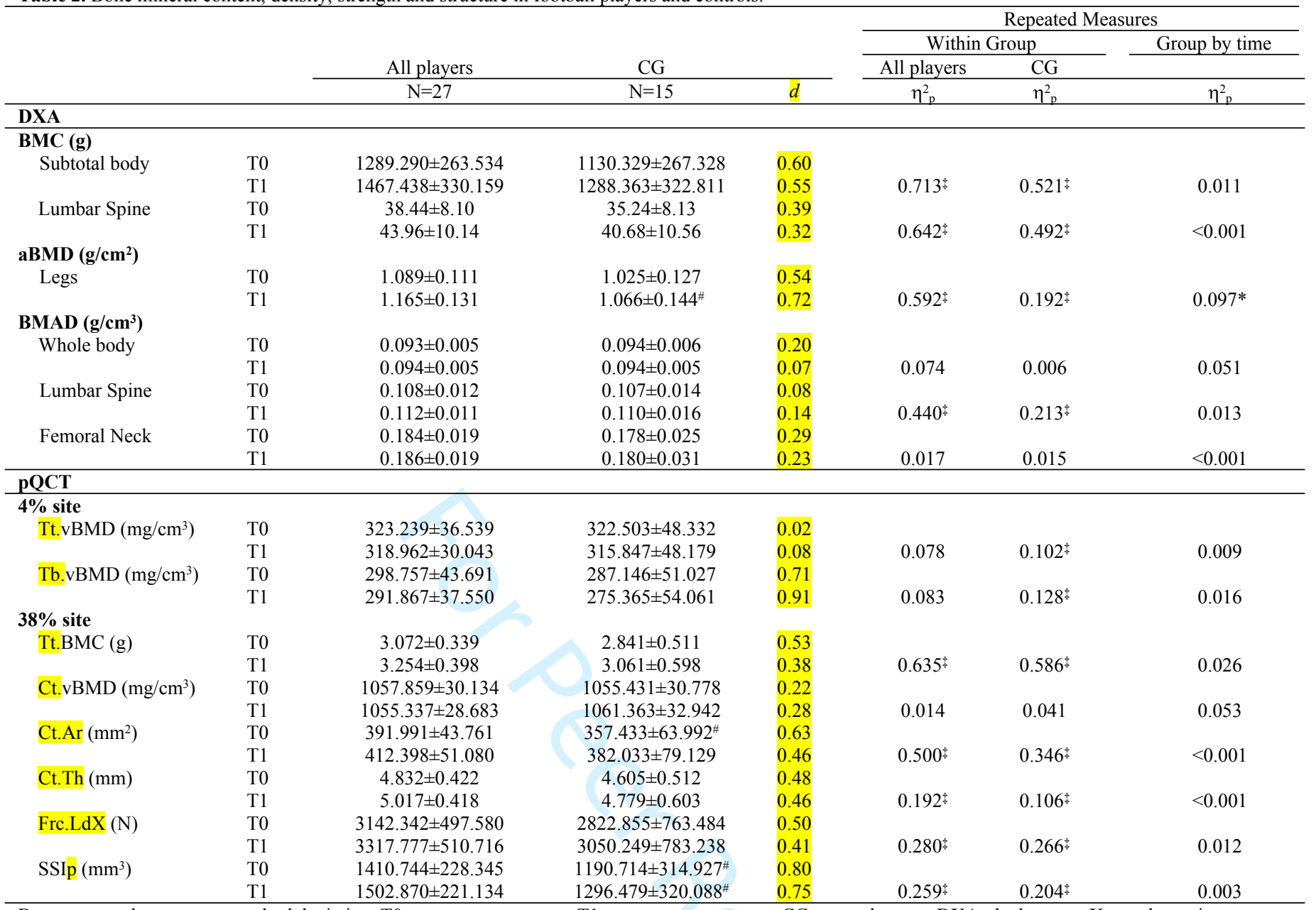

Data presented as mean \pm standard deviation. T0: pre-season moment; T1: post-season moment; CG: control group; DXA: dual-energy X-ray absorptiometry; BMC: bone mineral content; aBMD: areal bone mineral density; BMAD: bone mineral apparent density; pQCT: peripheral quantitative computed tomography; Tt.vBMD: total volumetric bone mineral density; Tb.vBMD: trabecular volumetric bone mineral density; Tt.BMC: total bone mineral content; Ct.vBMD: cortical volumetric bone mineral density; Ct.Ar: cortical cross sectional area; Frc.LdX: fracture load (axe X); SSIp: polar strain index; $d$ : Cohen`s $d$; $\eta^{2}$ : partial eta square.

\#Significant differences when compared to all players; ${ }^{\star}$ significant differences within groups between the beginning and the final of the season; *significant group by time interaction.

Cohen's $d$ can be small $(0.2-0.5)$, medium $(0.5-0.8)$ or large $(>0.8)$ and $\eta_{p}^{2}$ can be small $(0.01-0.06)$. medium $(0.06-0.14)$ or large $(>0.14)$. 
Table 3. Bone mineral content, density, strength and structure in football players who played in third-generation artificial turf with and without elastic layer.

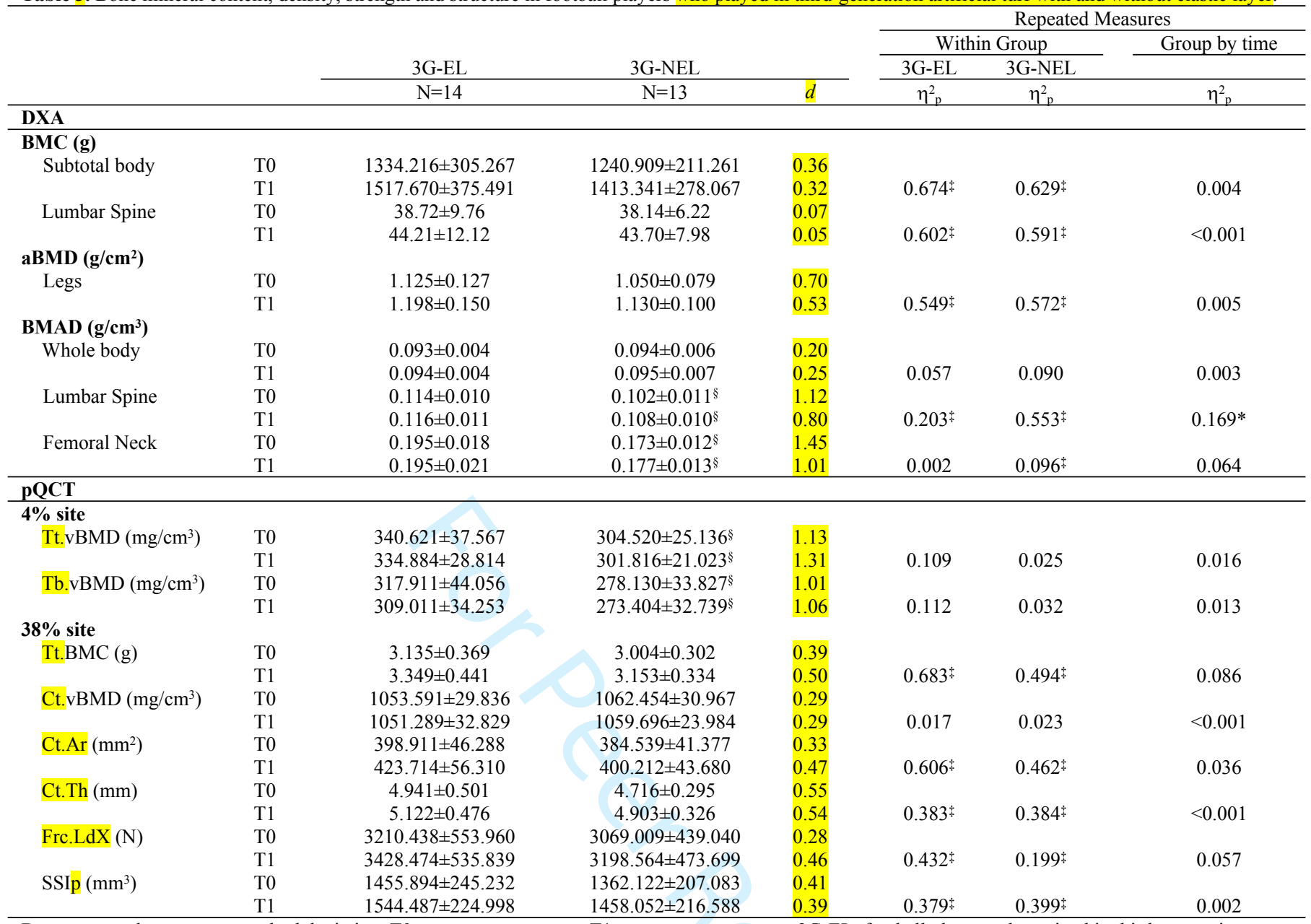

Data presented as mean \pm standard deviation. T0: pre-season moment; T1: post-season moment; 3G-EL: football players who trained in third-generation artificial turf with elastic layer; 3G-NEL: football players who trained in third-generation artificial turf without elastic layer; DXA: dual-energy X-ray absorptiometry; BMC: bone mineral content; aBMD: areal bone mineral density; BMAD: bone mineral apparent density; pQCT: peripheral quantitative computed tomography; Tt.vBMD: total volumetric bone mineral density; Tb.vBMD: trabecular volumetric bone mineral density; Tt.BMC: total bone mineral content; Ct.vBMD: cortical volumetric bone mineral density; Ct.Ar: cortical cross sectional area; Frc.LdX: fracture load (axe X); SSIp: polar strain index; $d$ : Cohen`s $d ; \eta_{\mathrm{p}}^{2}$ : partial eta square.

${ }^{\S}$ Significant differences when compared to 3G-EL; ;significant differences within groups between the beginning and the final of the season; *significant group by time interaction.

Cohen's $d$ can be small $(0.2-0.5)$, medium $(0.5-0.8)$ or large $(>0.8)$ and $\eta_{p}^{2}$ can be small $(0.01-0.06)$. medium $(0.06-0.14)$ or large $(>0.14)$. 
35 players of two football clubs and 62 students of two high schools were invited to participate

DATA AVAILABLE FOR ANALYSIS:

-Football players $(n=27)$

-Controls $(n=15)$

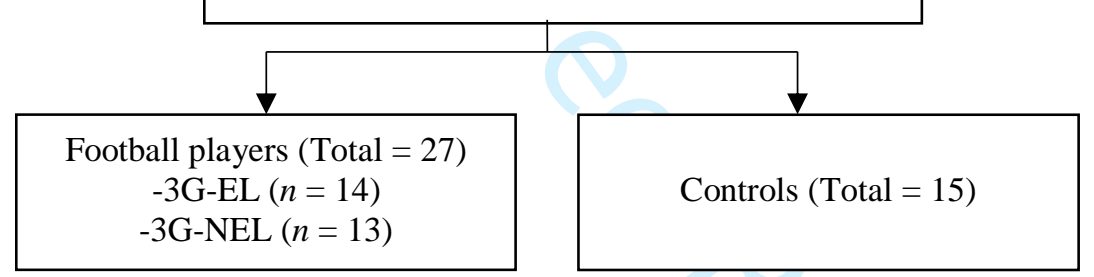



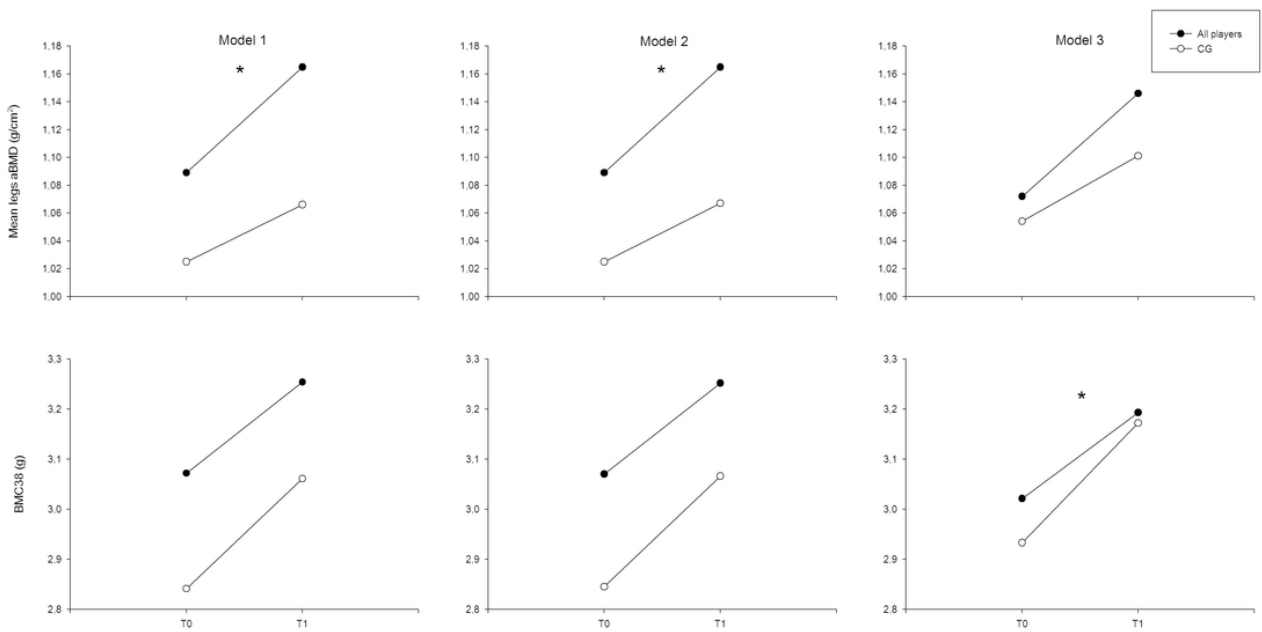

Figure 2 Legs aBMD and BMC38 interactions in football players and CG.

aBMD: areal bone mineral density; BMC38: total bone mineral content at the $38 \%$ of the length of the tibia; CG: control group; T0: pre-season moment; T1: post-season moment; Model 1: unadjusted data; Model 2: adjusted data by MVPA; Model 3: adjusted data by MVPA and subtotal lean mass (legs aBMD)/tibia muscle area (BMC38). *: Significant interactions were set at $\mathrm{p}<.05$.

$95 \times 48 \mathrm{~mm}(300 \times 300 \mathrm{DPI})$ 

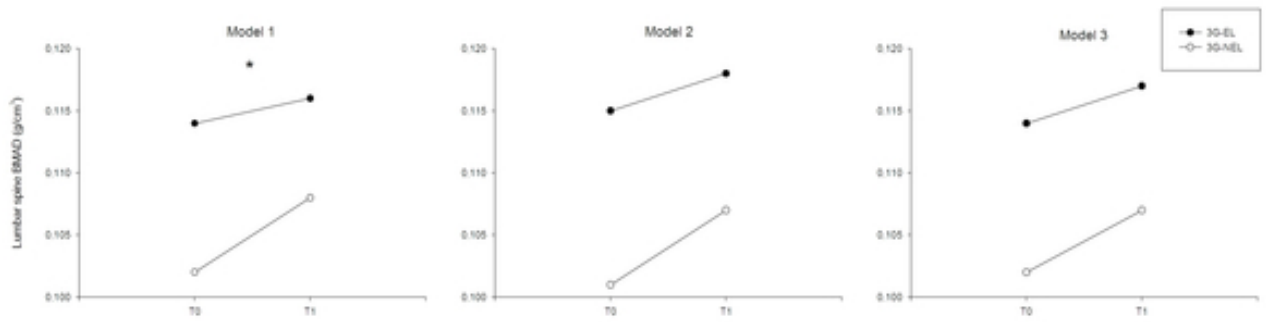

Figure 3 Lumbar spine BMAD interactions in football players.

BMAD: bone mineral apparent density; 3G-EL: football players who trained in third-generation artificial turf with elastic layer; 3G-NEL: football players who trained in third-generation artificial turf without elastic layer; T0: pre-season moment; T1: post-season moment; Model 1: unadjusted data; Model 2: adjusted data by MVPA; Model 3: adjusted data by MVPA and subtotal lean mass. *: Significant interactions were set at $\mathrm{p}<.05$.

$50 \times 13 \mathrm{~mm}(300 \times 300 \mathrm{DPI})$ 